\title{
Calcified Abdominal Pregnancy with Five Years of Evolution (Lithopedion): A Case Report
}

\author{
Nafisa A. Mohamed, Ikram A. Hajji, Salah A. Omar, Khalid S. Kalib, Abdirizak H. Ali \\ Department of Obstetrics and Gynecology, Benadir Hospital, Mogadishu, Somalia \\ Email: nafiso xaaji@hotmail.com
}

Received 12 June 2016; accepted 25 July 2016; published 28 July 2016

Copyright ( 2016 by authors and Scientific Research Publishing Inc.

This work is licensed under the Creative Commons Attribution International License (CC BY).

http://creativecommons.org/licenses/by/4.0/

c) (i) Open Access

\begin{abstract}
Lithopedion is a rare phenomenon resulting from an extra-uterine pregnancy that advances to fetal demise and calcification and there are less than 300 cases reported in 400 years of medical literature. This rare condition was first described by a surgeon of the Arabic era of medicine in the $10^{\text {th }}$ century. This case report is a 26-year-old, multiparous woman who had presented a lower abdominal pain for long time and she had never attended in pre-natal clinic. She came to our hospital with pain and tumoral mass in infra-umbilical area and then we referred to radiology center, after that ultrasound examination of radiology center demonstrated an extra-uterine abdominal 30-week pregnancy measuring the femoral length, this for first diagnosis of radiology center. After laparotomy was performed we met an oval shaped mass that attached the omentum in peritoneal cavity and this mass was a fetus retention of 5 years without calcified ovular membranes but the fetus was calcified so this type is called lithopedion that describes according to the Kuechenmeister classification in 1881 . She made good post-operative recovery after extraction the stone baby.
\end{abstract}

\section{Keywords}

Abdominal Pregnancy, Lithopedion

\section{Introduction}

An abdominal pregnancy represents a variant of an ectopic pregnancy, where it formed outside of the uterus, and it resides within the peritoneal cavity exclusive of tubal, ovarian and intra-ligamentous sites of implantation, while the placenta is often attached to multiple locations such as bowel, omentum, uterine cul-de-sac and pelvic 
sidewall [1]. The abdominal pregnancy has a very high morbidity and mortality for both mother and fetus ( $0 \%$ $20 \%)$ and (40\% - 95\%) respectively [1] [2]. The incidence of abdominal pregnancy is 1:11,000 births and 1:100 in ectopic gestations while it also varies widely with geographical degree of antenatal attendance and socioeconomic status [1] [3] [4]. The diagnosis of abdominal pregnancy is difficult especially in low resource centers. The forms beyond fifth month are more common in developing countries. Early diagnosis of abdominal pregnancy can reduce the maternal risk [5]. If the abdominal pregnancy remains undiagnostic and untreated followed by fetus demise, lithopedion forms; that is calcification of fetal parts and surrounding membranes in an expected outcome. Lithopedion is the word from Greek which means litho = stone and pedion = child and it is a rare form of abdominal pregnancy which occurs 1.5\% - 1.8\% [1] [6]. Although, the incidence of lithopedion of all pregnancy is remarkably less which is $0.00054 \%$, less than 300 cases have been published in 400 years in the literature of the world [3] [6] [7]. The duration of holding of the dead fetus varies between 4 and 70 years but in our case the durations is 5 years; and the patients' age ranges from 23 to 100 years, while in our case the age is 26 years [8]. We report here a case of advanced extra-uterine abdominal pregnancy (lithopedion) confirmed by ultrasonography, the presence of calcified fetus and investing membranes with gestational age of 30 weeks, while the period of lithopedion holding was estimated to be about 5 years.

\section{Observation}

A 26-year-old woman was referred to our department of obstetrics and gynecology at Benadir Hospital, Mogadishu-Somalia with complained of a lower abdominal pain with associated palpitation, dizziness, poor appetite and insomnia for the last seven months. She started menarche at age of 15 years and she was married at age of 17 years old. Her obstetrical and gynecological history was normal such as menstrual cycle 28 days and its regular for every month with length of menstrual period is 4 to 5 days even after pregnancy the mensural bleeding was started. She denied to take any contraceptive drugs. Her personal and family history was unremarkable. She was a multiparous woman. The first baby was delivered normally by traditional birth attendant (TBA) and her second pregnancy was also normal and delivered by TBA. The third pregnancy was the diagnosis of now (Abdominal pregnancy) but she didn't get any medical checkup and the patient reported during eight month was started vaginal bleeding which is not enormous and spontaneously stopped this bleeding, during this term (Infraumbilcal mass-that became Abdominal pregnancy) the mother was delivered three babies with normal delivery at traditional birth attendant but the last delivery was developed post-partum hemorrhage (PPH) and she did not receive any pre-natal follow-up. On examination was revealed infra-umbilical mass with mobile and hardened (Figure 1). Laboratory findings were within normal limits but in full blood count (Low Leucocytes, Erythrocytes and Hemoglobin). An ultrasonography was obtained and it clearly showed an empty uterus, regular ovaries and the presence of an abdominal 30 weeks fetus measuring femur length (Abdominal pregnancy) for first diagnosis. After laparotomy a fetus within sac or an oval tumor was seen in the peritoneal cavity in adhesion with

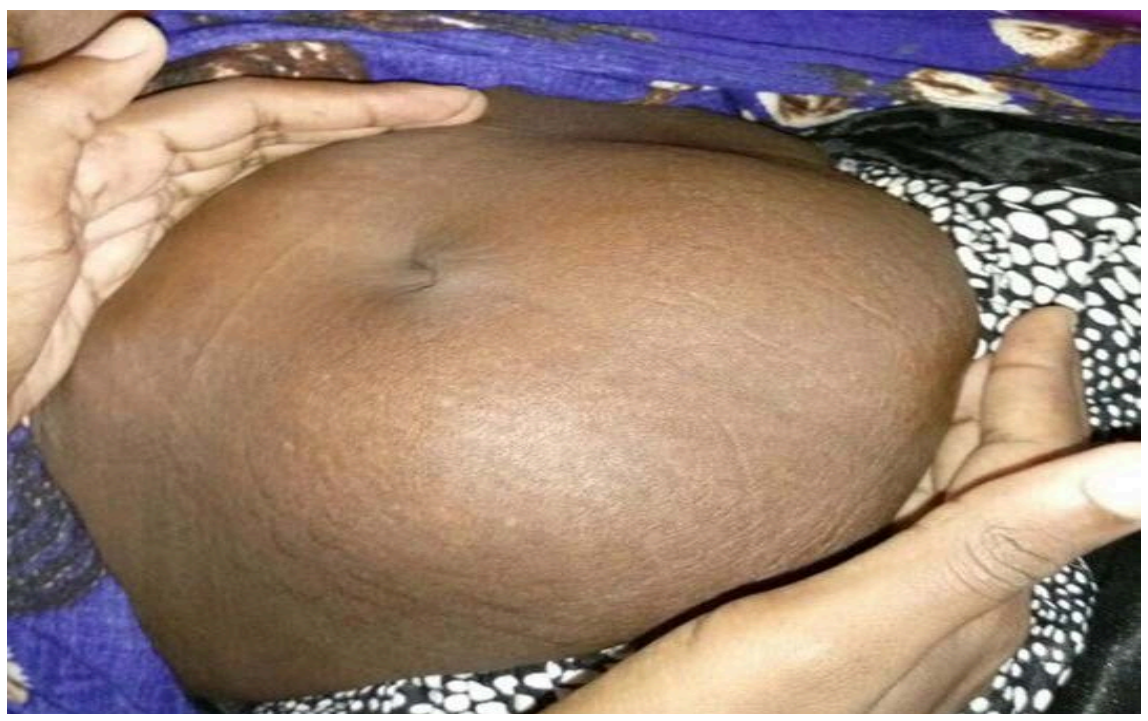

Figure 1. Infra-umbilical mass. 
omentum (Figure 2, Figure 3). After dissection of the sac whole fetus with ossified skeleton was found. The fetus was intact the amniotic cavity without amniotic fluid (Calcified Abdominal Pregnancy-Lithopedion) (Figure 4) and this was the second diagnosis after the sonography.

\section{Discussion}

Lithopedion is a term derived from the Greek words which means Litho (Stone) and Paedion (child) and it explain an extra-uterine dead fetus that has developed calcified. It is a rare condition that was first described in the tenth century by Albucasis, a surgeon of the Arabic era of medicine [9]. There are some conditions contribute

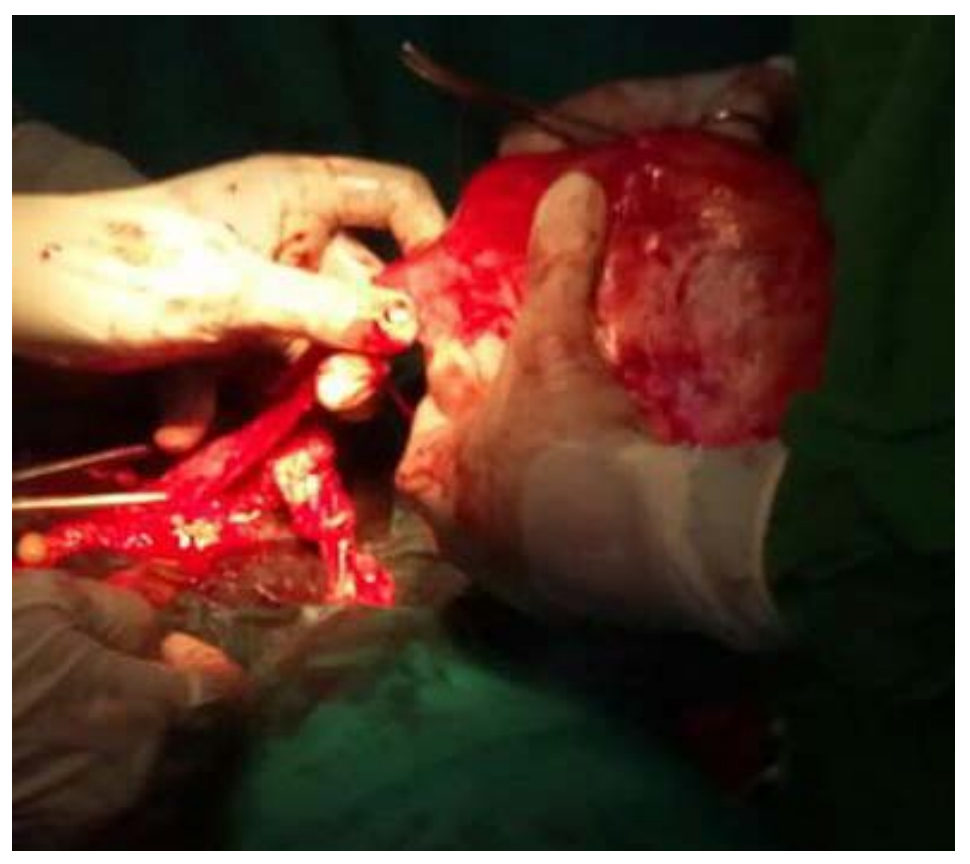

Figure 2. Calcified abdominal pregnancy-peritonium adherence.

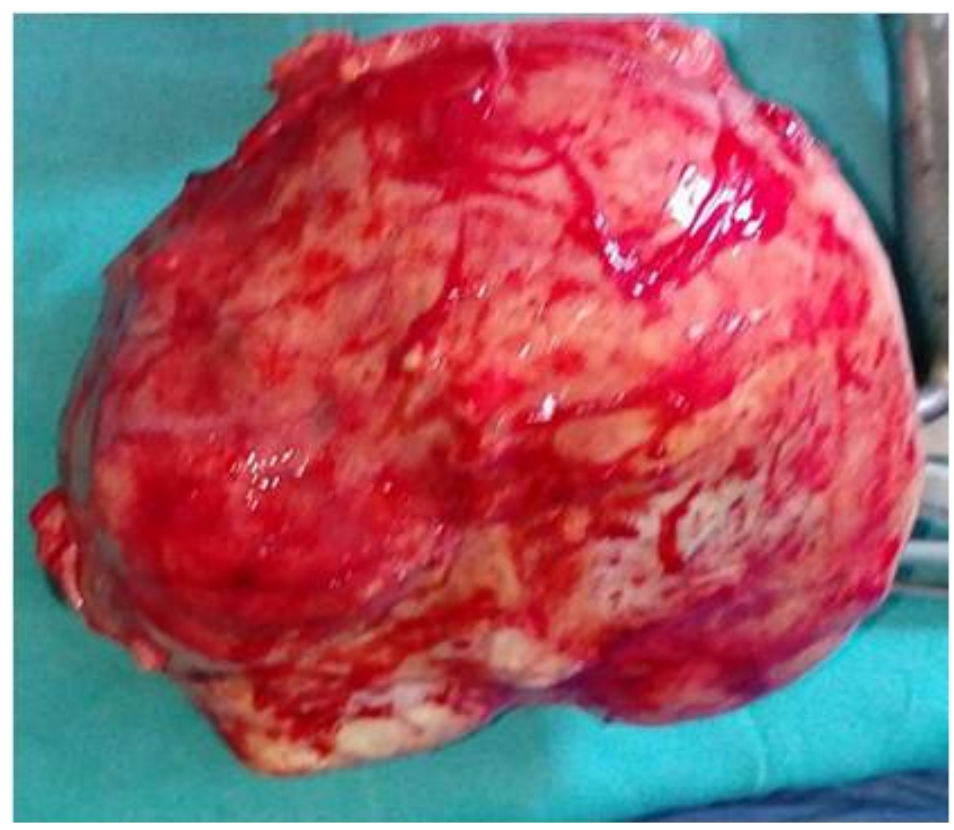

Figure 3. Head, hand and back are clearly identified. 


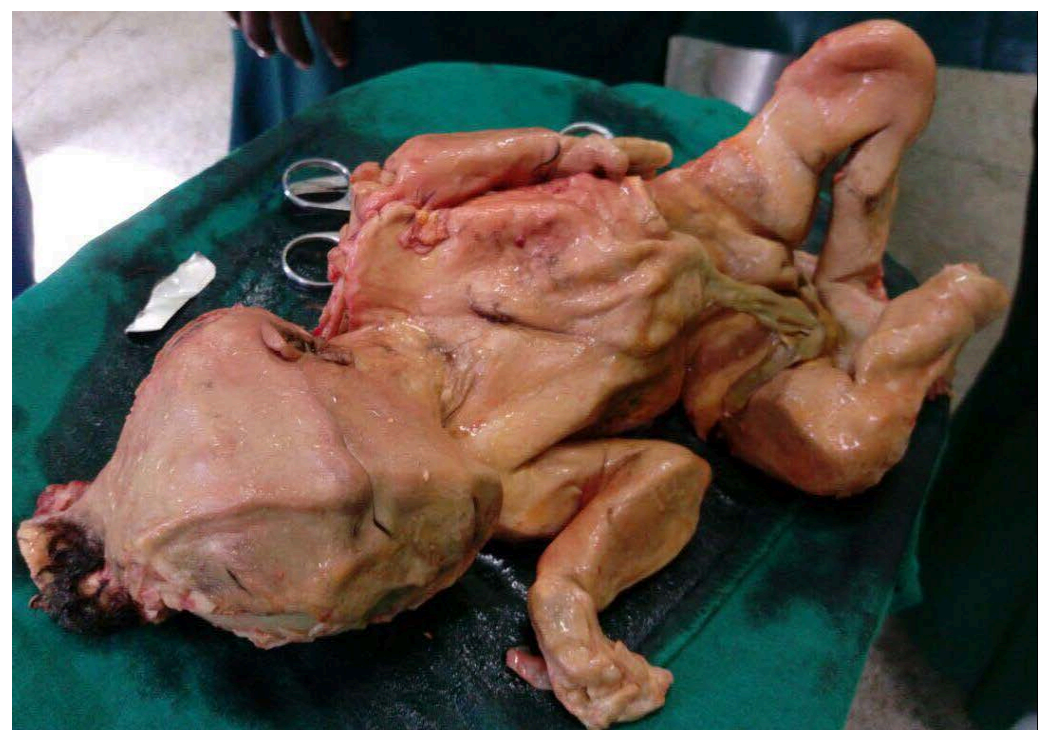

Figure 4. Calcified abdominal pregnancy-lithopedion after dissection of uncalcified ovular membrane.

the formation of lithopedion: 1) extra-uterine pregnancy; 2) fetal death after 3 months of pregnancy; 3) the egg must be sterile; 4) difficult diagnosis and missing in early diagnosis; 5) deposition of calcium in the local conditions. So this pregnancy is same as for abdominal intra-uterine pregnancy in terms of the development until fetal death. Later on dehydration of tissue and calcium infiltration occur [1] [3] [7]. In 1881 Kuechenmeister, genetically this formation is called lithopedion and it divided into three form: 1) lithokelyphos which means litho = rock, kelyphos = shell and it is the ovular membrane is calcified and the fetus can be in different stages of decomposition; 2) lithokelyphopedion which both of fetus and ovular membrane were calcified; the last term is lithopedion which is only the fetus is calcified as in our case now [8] [9].

This case highlights the need to maintain a high index of suspicion for this rare and serious condition, particularly if presented with unusual clinical (e.g. recurrent abdominal pain, fetal malpresentation) and ultrasound appearances. In fact the clinical history, physical examination, laboratory and ultrasonographic findings frequently vary in abdominal pregnancy.

In the literature of this case, the age of the patients was varies and the ranged from 23 to 100 years old in the related cases, two-third of them were being over 40 years old. The period of fetal retention was varied the case were reported and it is from 4 to 70 years [8], but in our case the period of the fetus retention was 5 years.

The lithopedion cases are discovered by incidentally at surgery, autopsy or on Imaging studies [9]. In abdominal pregnancies are and it's usually secondary to tubal rupture or tubal abortions. There is a consequent of reimplantations of the embryo onto bowel, omentum or mesentery, in our case the re-implantation was formed in omentum [10] [11]. The factors that contribute for ectopic pregnancy in general are congenital anomalies, endometriosis, previous pelvic infection, pervious ectopic pregnancy and tubal surgery [11].

The consequents of lithopedion are intestinal obstruction, caecal volvulus and perforation, abscess formation of urinary bladder and rectum as well as fetal parts penetration through the wall [10].

Mortality abdominal pregnancy was higher than the tubal pregnancy and intra-utreine pregnancy, due to intraabdominal bleeding which leads to anemia, infections, disseminated intravascular coagulopathy, pulmonary embolism and fistulae that caused by infiltration of fetal bones [12].

Imaging studies was sufficient such as abdominal X-ray, computed tomography (CT-Scan) and magnetic resonance imaging (MRI) to show clearly, and the most cases was showed by ultrasound but there is a missing by the operator or the skill of the operator and it's enough to confirm the diagnosis. The differential diagnosis considered by other disease such as teratomas, ovarian tumors, calcified uterine fibroids, inflammatory masses, urinary tract and bladder tumors.

There are some cases retained abdominal pregnancy was reported without surgical intervention due to possibility of complications, but even the years the proper procedure is surgical removal and there are other cases 
may need early surgical intervention after the consideration [10]. We recommended in the while in Operation Theater; two of one surgeon would be recommended either general surgeon or urologist with their help due to the possibilities of large quantities of abdominal vessels and intestinal adherence in our case we have general surgeon.

\section{Conclusion}

The intra-abdominal pregnancy is a rare and a serious condition with significant maternal and perinatal mortality in which problem of the diagnosis of the disease was missing during antenatal care. Good history taking and physical examination are important in this case, and also the imaging studies such as X-rays, repeated ultrasound, CT-scan and MRI help settle the diagnosis of this disease. Due to the absence of adequate medical attention for clinicians and health workers, we suggest that the clinicians should be attentive in diagnosis of this disease; once he suspects, we recommend asking for help from the experienced clinicians.

\section{Acknowledgements}

We are thankful to Mohamed A. Hassan-Kadle for helping us during preparation of the manuscript.

\section{References}

[1] Costa, S.D., Presley, J. and Bastert, G. (1991) Advanced Abdominal Pregnancy. Obstetrical \& Gynecological Survey, 46, 515-525. http://dx.doi.org/10.1097/00006254-199108000-00003

[2] Berghella, V. and Wolf, S.C. (1996) Does Primary Omental Pregnancy Exist? Gynecologic and Obstetric Investigation, 42, 133-136. http://dx.doi.org/10.1159/000291922

[3] Irick, M.B., Kitsos, C.N. and O’Leary, J.A. (1970) Therapeutic Aspects in the Management of a Lithopaedion. American Journal of Surgery, 36, 232-234.

[4] Atrash, H.K., Friede, A. and Hogue, C.J. (1987) Abdominal Pregnancy in the United States: Frequency and Maternal Mortality. Obstetrics \& Gynecology, 69, 333-337.

[5] Achenani, M., Kouach, J., et al. (2014) Abdominal Pregnancy: Case Report. Science Journal of Clinical Medicine, 3, 17-20.

[6] Frayer, C.A. and Hibbert, M.L. (1999) Abdominal Pregnancy in a 67 Year Old Woman Undetected for 37 Years: A Cases Report. Journal of Reproductive Medicine, 44, 633-635.

[7] Spiritos, N.M., Eisenkop, S.M. and Mishell, D.R. (1987) Lithokelyphos: A Case Report and Literature Review. Journal of Reproductive Medicine, 32, 43-46.

[8] Passini, J.R., Roxana, K., Angela, P.M., et al. (2000) Calcified Abdominal Pregnancy with Eighteen Years of Evolution: Case Report. São Paulo Medical Journal-Revista Paulista de Medicina, 118, 192-194. http://dx.doi.org/10.1590/s1516-31802000000600008

[9] Lachman, N., Satyapal, K.S., Kalideen, J.M. and Moodley, T.R. (2001) Lithopedion: A Case Report. Clinical Anatomy, 14, 52-54. http://dx.doi.org/10.1002/1098-2353(200101)14:1<52::AID-CA1009>3.0.CO;2-H

[10] Kim, S.M., Park, S. and Lee, T.S. (2002) Old Abdominal Pregnancy Presenting as an Ovarian Neoplasm. Journal of Korean Medical Science, 17, 274-275. http://dx.doi.org/10.3346/jkms.2002.17.2.274

[11] Cotter, A., Izquierdo, L. and Heredia, F. (2000) Abdominal Pregnancy.

[12] Kun, K.Y., Wong, P.Y., Ho, M.W., et al. (2000) Abdominal Pregnancy Presenting as a Missed Abortion at 16 Weeks' Gestation. Hong Kong Medical Journal, 6, 425-427. 


\section{Submit or recommend next manuscript to SCIRP and we will provide best service for you:}

Accepting pre-submission inquiries through Email, Facebook, LinkedIn, Twitter, etc.

A wide selection of journals (inclusive of 9 subjects, more than 200 journals)

Providing 24-hour high-quality service

User-friendly online submission system

Fair and swift peer-review system

Efficient typesetting and proofreading procedure

Display of the result of downloads and visits, as well as the number of cited articles

Maximum dissemination of your research work

Submit your manuscript at: http://papersubmission.scirp.org/ 